\title{
An Introduction to Alternative Experimental Models in Monitoring Economic Failures
}

\author{
Mario Arturo Ruiz Estrada', Muhammad Tahir ${ }^{2}$
}

\begin{abstract}
This research paper presents four alternative experimental models for monitoring economic failures. These models include the national production function (NP-Function), the multi-level trade creation and trade diversion analytical framework, the mega-economic structures vulnerability analysis (MSV-Analysis), and the mega-economic surface interactive system. The main objective behind the proposed experimental models in economics is to analyze different macroeconomic scenarios in monitoring and warning of possible unexpected economic failure(s) under the use of a new mathematical framework and the application of multidimensional graphs. The proposed alternative experimental models are based on the application of Econographicology. Hence, our models are expected to offer policy-makers and researchers new analytical tools to study the impact and trend of economic failures in the economy of any country from a new perspective. Finally, we suggest the application of three new concepts in the study of economic failures such as Omnia Mobilis (everything is moving), the global structural imbalance principle, and the dynamic imbalance state. Therefore, our findings would certainly help both policy makers and researchers to implement and execute appropriate policies to develop their economies and to protect them from unwanted situations.
\end{abstract}

KEY WORDS: $\quad$ Econographicology, Economic Modeling, Macroeconomic Policy, Economic Teaching, Multi-Dimensional graphs and Multi-Dimensional Physical Spaces

JEL Classification: E60

${ }^{1}$ Department of Economics, Faculty of Economics and Administration, University of Malaya, Malaysia; ${ }^{2}$ Department of Management Sciences, Comsats Institute of Information Technology, Abbottabad, Pakistan

\section{The National Production Function (NP-Function)}

\subsection{Introduction}

It is well understood from the study of the Cobb 正

Douglas production function that the use and com-

Correspondence concerning this article should be addressed to: Mario Arturo Ruiz Estrada, Department of Economics, Faculty of Economics and Administration, University of Malaya, Kuala Lumpur 50603, T: (+60) 37967-3728. E-mail: marioruiz@um.edu.my bination of labor (L) and capital $(\mathrm{K})$ variables are not sufficient to quantify and analyze final output (Y). In fact, the NP-Function includes labor (L) and capital (K) plus " $n$ " number of variables together in its analysis. The NP-Function is basically formed by four sub-production functions where each sub-production function stands for the production of various sectors of the economy such as agriculture, industry, manufacturing and services (Schlicht, 1985). The same NPFunction is based on the use of an economic real time modeling framework that is based on the application 
of dynamic growth rates. It should be noted that all variables included in each sub-production function are growth rates. We assume that the sub-production functions in the NP-Function remains in a constant dynamic imbalance state (Ruiz Estrada, 2008). It is to explain and analyze the behavior of complex dynamic changes of each sub-production function until the final national output is achieved. We propose however, the construction of the NP-Function under the application of Econographicology (Ruiz Estrada, 2007) and an economic real time modeling framework. The NP-Function can facilitate the analysis and visualization of the sub-production functions of the production sector such as the agriculture, industry, manufacturing and services that maintain constant movement in real time in the same physical space; it is to observe the fast and abrupt changes of the four sub-production functions by the production sector developing in real time. Finally, the NP-Function is available to offer an alternative economic model to measure the final national output.

\subsection{The National Production Function (NP- Function)}

This section explains the construction of the national production function. Initially, the NP-Function is built of four sub-production functions where each sub-production function has its own quadrant. Each quadrant shows a single dependent variable represented by a vertical line in the central part of its quadrant by $\mathrm{Y}_{\mathrm{ij}}$ and " $\mathrm{n}$ " number of independent variables $\mathrm{X}_{\mathrm{ij}}$ on the bottom part of the quadrant by horizontal lines. Finally, we join all $\mathrm{X}_{\mathrm{ji}}$ to the dependent variable $\mathrm{Y}_{\mathrm{ij}}$ under the application of “ $\overline{\mathrm{T}}$ " linkage of axes. All axes in each quadrant are running on real time under the application of dynamic growth rates (see expression 5). Therefore, we have four quadrants or four sub-production functions where each quadrant has its dependent variable " $Y_{i j}$ " and " $n$ " number of independent variables " $\mathrm{X}_{\mathrm{ij}}$ ", respectively. Similarly, we have four outputs from the agriculture sector $\left(\mathrm{Y}_{0}\right)$, industrial sector $\left(\mathrm{Y}_{1}\right)$, manufacturing sector $\left(\mathrm{Y}_{2}\right)$ and services sector $\left(\mathrm{Y}_{3}\right)$ from each sub-production function. Finally, we can observe that among the four quadrants there exists a single axis that we call the final national output " $\mathrm{Y}^{*}$ ". It is obtained by joining the four outputs from each sub-production sector by the application of the “吉” linkage of quadrants by straight lines (see Figure 1). The idea is to build a single surface based on the links of four outputs together in the same physical space. In the NP-Function, our objective is to build a large and single surface that is moving in real time in the same physical space. In fact, the application of the Omnia Mobilis assumption (Ruiz Estrada, 2011) is the basic condition to generate the real time effect of the NP-Function. Hence, the final national output " $\mathrm{Y}$ " always remains dynamic with multi-dimensional behavior according to the economic modeling in real time in its multi-dimensional space (Ruiz Estrada, 2013). Finally, the analysis of the result of the NP-Function depends on the position of the surface, which can help to determine the situation of any economy. Additionally, the NP-Function result will be determined by the position of the surface. If the surface is located at a positive level, then we can observe economic stability (see Expression 7). If the surface remains at a zero level, then we can observe economic stagnation (see Expression 8). If the surface remains at either negative or positive levels, then it is possible to observe economic recession (see Expression 9). Finally, if the surface is located on the negative level then we find an economic crisis (see Expression 10).

\subsection{Model}

The NP-Function offers an alternative graphical and mathematical modeling approach to analyzing the final national output from a multi-dimensional perspective. This new multi-dimensional graphical modeling can show the final national output. The NP-Function provides to policy makers an alternative methodological approach to measure the final output of any country from the national production function. The NP-Function is followed by four sub-production functions, which are as follows:

Sub-Production Function 0:

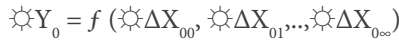

Sub-Production Function 1:

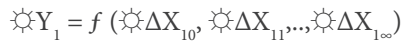

Sub-Production Function 2:

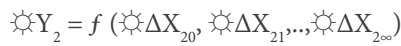




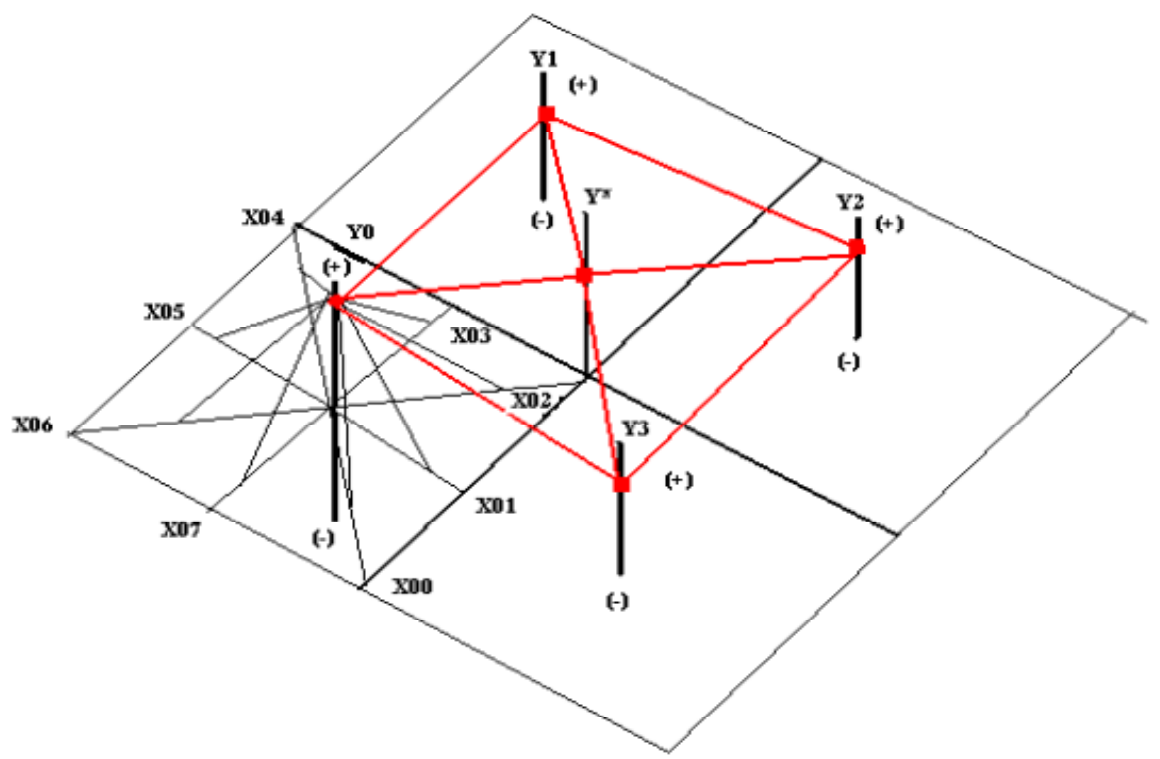

Figure 1. The National Production Function (NP-Function)

Sub-Production Function 3:

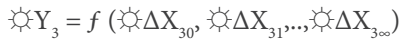

Real Time $\mathrm{Y}_{\mathrm{i}}=$ Output $\Delta=$ Dynamic Growth Rate

All variables in each sub-production function request the application of the dynamic growth rate follow

$\Delta \mathrm{X}_{\mathrm{ij}}=\Delta \mathrm{X}_{\mathrm{ij}\langle\mathrm{t}+1>}-\Delta \mathrm{X}_{\mathrm{ij}<t 0>} \times 100 \%=>\Delta \mathrm{X}_{\mathrm{ij}<\mathrm{t} 0>}$

$\mathrm{i}=\{1,2, \ldots, \infty\}$ and $\mathrm{j}=\{1,2, \ldots \infty\}<\mathrm{t}_{+1}>=$ Future period of time $<\mathrm{t}_{\mathrm{o}}>=$ Initial period of time.

Therefore, the final mathematic structure to build the NP-Function is equal to expression (6).

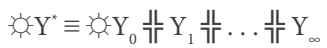

少= linkage of quadrants.

The NP-function result will depend on the location of the surface (see Figure 1). Hence, we have four pos- sible results that can be classified by Economic Stability (see Expression 7), Economic Stagnation (see Expression 8), Economic Recession (see Expression 9) and Economic Crisis (see Expression 10) (Barro, 1976 and Hansen, 1938):

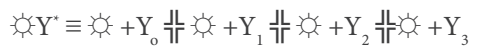
$\left\{\right.$ if $\Delta+\mathrm{Y}^{*} \cap \mathrm{R}_{+}$then the surface $\equiv$ Economic Stability $\}$

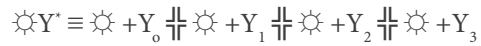
$\left\{\right.$ if $\Delta Y^{*} \cap 0$ then the surface $\equiv$ Economic Stagnation $\}$

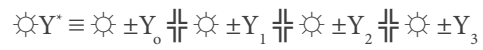
if $\Delta \mathrm{Y}^{\star} \cap \mathrm{R}_{+/-}$then the surface $\equiv$ Economic Recession Alert\}

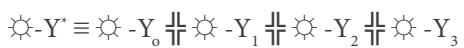
$\left\{\right.$ if $\Delta \mathrm{Y}^{*} \cap \mathrm{R}$ then the surface $\equiv$ Economic Crisis $\}$ 


\section{Multi-level Trade Creation and Trade Diversion Analysis}

\subsection{Introduction}

Economists and policy makers have employed a variety of analytical tools to study the behavior of the external sector in different countries and regions since Smith (1776) and Ricardo (1819). The most common analytical tools applied in such studies so far are the terms of trade (ToT) used by Balassa (1985) and the openness index (O) included by Edwards (1993) in his paper titled "Openness, Productivity and Growth: What Do We Really Know?". We introduce in this paper a complementary set of indicators to evaluate trade creation and trade diversion from different perspectives. We name these indices the trade mass $(\mathrm{T})$, the intra-regional trade trend ratio $\left(\mathrm{R}_{1}\right)$, the extra-regional and trade trends ratio $\left(\mathrm{R}_{2}\right)$ and the trade expansion coefficient (TEC).

\subsection{Introduction to the Trade Mass (T)}

The main idea behind building the trade mass $(\mathrm{T})$ index is to study the trade volume behavior of any country by the application of the sphere volume. The inspirational idea behind using the sphere volume is that it can help to make the process of visualization of the trade volume behavior graphically easier. Hence, the trade mass $(\mathrm{T})$ is can be observed graphically from a single sphere. This single sphere would take different sizes across different periods of time and spaces. In fact, the trade mass (T) follows the application of the classic formula of the volume of a sphere denoted by expression 1 as mentioned in the research of Russell (1897). Hence, the volume of a sphere can be obtained by multiplying $4 / 3$ and $\pi$ and the radius squared. $\pi$ is a well-known mathematical constant whose value is approximately equal to 3.1416 .

$V=(4 / 3) \pi r^{2}$

In expression 3, the trade mass $(\mathrm{T})$ is constructed keeping $\pi$ as constant and the radius is fixed by the growth rate of the trade volume $(\Delta \mathrm{T})$. The growth of trade volume is calculated from the trade volume growth rate between the previous year ( $\mathrm{t}-1)$ and the current year (t) as described in expression 2.
$\Delta \mathrm{T}=(\mathrm{X}+\mathrm{M})_{\mathrm{t}}-(\mathrm{X}+\mathrm{M})_{\mathrm{t}-1} /(\mathrm{X}+\mathrm{M})_{\mathrm{t}-1}$

$\mathrm{T}=(4 / 3) \pi(\Delta \mathrm{T})^{2}$

Finally, the analysis of the trade mass (T) depends on the size of the volume in different spheres across different periods of time and space. The key variable that establishes the size of the sphere volume is the growth rate of trade volume as shown by $(\Delta \mathrm{T})$. We find that differences in the size of spheres are helpful in observing whether there exists expansion, contraction or stagnation of the trade volume of any country across different periods of time and space. We would like, however, to recommend the use of different colors for the different spheres. It could help to visualize easily the behavior of the trade mass $(\mathrm{T})$ in the same graphical space. The difference between the trade volume histogram in 2 dimensions and the trade mass $(\mathrm{T})$ is that we are able to observe the size of any country from a different graphical perspective (Inselberg and Dimsdale, 1994). Additionally, the advantage of using the trade mass $(\mathrm{T})$ is also to observe the process of accumulation from a long historical data of the trade volumes in different periods of time under the visualization of a longer structure formed by joining a large number of spheres together in the same graphical space (see Figure 2). This large structure is entitled "The Trade Accumulation Structure -TES-". Therefore, we can observe clearly the expansion, contraction or stagnation of each trade mass $(\mathrm{T})$ in the large string of spheres across different periods of time and space. It can give us a multidimensional effect to observe the behavior of the international trade in any country.

\subsection{Multi-level Trade Creation and Trade} Diversion Analysis

The multi-level trade creation and trade diversion analysis starts with the building of the trade mass by an intra-regional level (Tir) and extra-regional level (Ter). The building of the trade mass using the intra-regional level (Tir) and extra-regional level (Ter) is dependent on the measurement of the growth rate of trade volume with the help of the intra-regional level $(\Delta$ Tir) and extra-regional level ( $\Delta$ Ter) as denoted by expression 4 and 5. Finally, we proceed to measure the trade mass by the intra-regional level (Tir) and extra-regional level (Ter) under the application of expressions 5 and 7. 


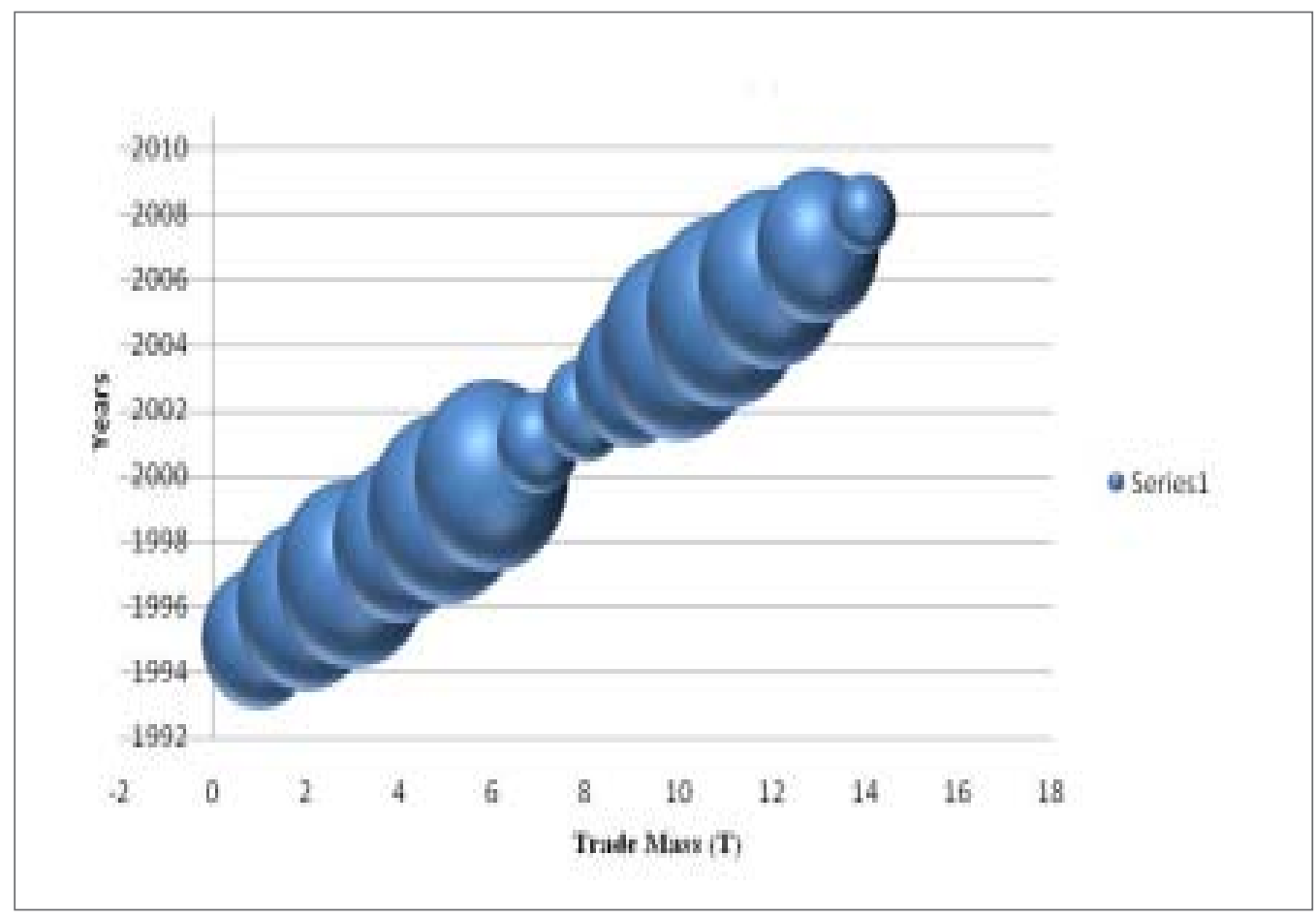

Figure 2. Trade Mass

$$
\begin{aligned}
& \Delta \text { Tir }=(\text { Xir }+ \text { Mir })_{\mathrm{t}}-(\text { Xir }+ \text { Mir })_{\mathrm{t}-1} /(\text { Xir }+ \text { Mir })_{\mathrm{t}-1} \\
& \text { Tir }=(4 / 3) \pi(\Delta \text { Tir })^{2} \\
& \Delta \text { Ter }=(\text { Xer }+ \text { Mer })_{\mathrm{t}}-(\text { Xer }+ \text { Mer })_{\mathrm{t}-1} /(\text { Xer }+ \text { Mer })_{\mathrm{t}-1}(6) \\
& \text { Ter }=(4 / 3) \pi(\Delta \text { Ter })^{2}
\end{aligned}
$$

The next task is to find the intra-regional $\left(\mathrm{R}_{1}\right)$ and extra-regional $\left(\mathrm{R}_{2}\right)$ trade trend ratios, respectively, with the help of expressions 8 and 9 . We are interested to remind note that the trade mass $(\mathrm{T})$, extra-regional and intra-regional, plays an important role in measuring the trade trend and trade creation and diversion effect of any economy. This is possible through the comparison of intra-regional $\left(\mathrm{R}_{1}\right)$ and extra-regional $\left(\mathrm{R}_{2}\right)$ trade trend ratios. There are two possible scenarios. If Tir is larger than the Ter, then we can observe that there exists trade creation under the intra-regional level but trade diversion in the extra-regional level. However, if Ter is greater than Tir, then we can witness evidence supporting trade creation under the extra-regional level and trade diversion in the intra-regional level.

$\mathrm{R}_{1}=$ Tir / Ter

$\mathrm{R}_{2}=\mathrm{Ter} / \mathrm{Tir}$

Finally, we want to find the trade expansion coefficient (TEC), which can be constructed by multiplying the intra-regional $\left(\mathrm{R}_{1}\right)$ and extra-regional $\left(\mathrm{R}_{2}\right)$ trade trend ratios, as seen from expression 10. As a consequence, we are able to find the trend of the behavior of international trade. The final outputs of the trade expansion coefficients (TEC) have two possible results. If the TEC is high, there exists the possibility of a fast trade volumes expansion. Similarly, if the TEC is low, there exists the possibility of a slow expansion of the trade volume. 

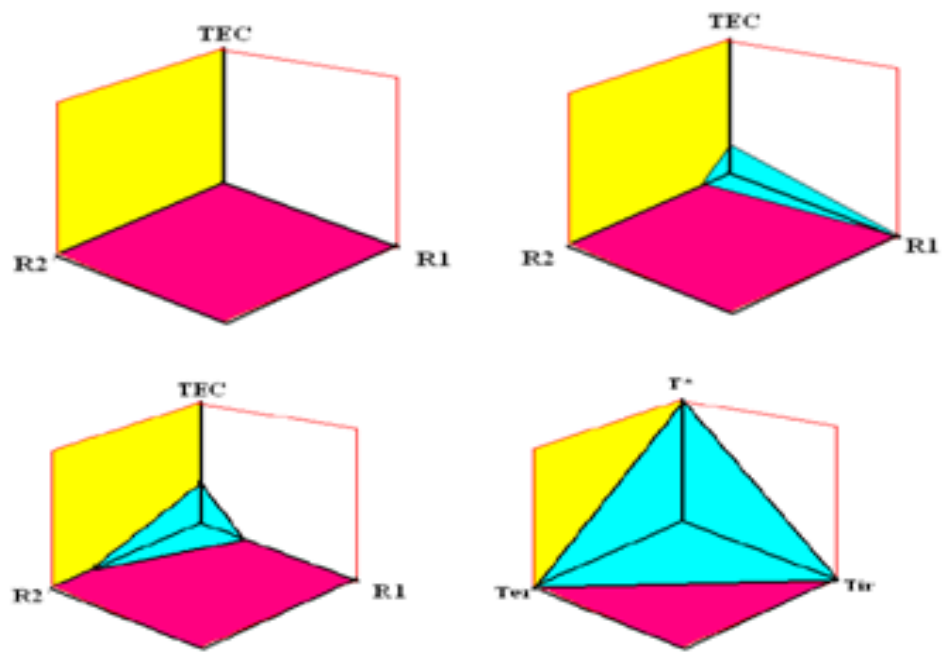

Figure 3. The Graphical Analysis of the Multi-Level Trade Creation and Diversion

$\mathrm{TEC}=\mathrm{R}_{1} * \mathrm{R}_{2}$

Graphically, the multi-level trade creation and trade diversion is based on the use of a 3-dimensional Cartesian plane. The plotting into the 3 -dimensional Cartesian plane (Brouwer, 1913) is divided using three axes; the $\mathrm{X}$-axis, represents the intra-regional trade trend ratio $\left(R_{1}\right)$, the $Y$-Axis represents the extraregional trade trend ratio $\left(R_{2}\right)$ and the trade expansion coefficient (TEC) is represented on the Z-axis. Figure 3 summarizes the entire process. Afterward, we proceed to join these three variables with the help of straight lines until we build a figure represented by a single pyramid (see the blue area in each 3 -D coordinate system). Finally, the graphical analysis of the multi-level trade creation and trade diversion depend on the behavior of the figure (pyramid), which can experience an expansion, contraction or stagnation at any time.

\section{Mega-economics Structures Vulnerability Analysis (MSV- Analysis)}

\subsection{Introduction}

The macroeconomics structures vulnerability analysis (MSV-Analysis) has two general objectives. The first objective is to evaluate the weaknesses and strengths of different macroeconomic structures (or scenarios) in the same graphical space simultaneously. The second general objective is to simultaneously forecast different macroeconomic structures. The MSV-Analysis is based on the application of the Cubes Cartesian Physical Space as documented in the novel work of Ruiz Estrada (2007). The Cubes-Cartesian physical space opens the possibility of generating a multi-dimensional visual effect to show the vulnerability of many macroeconomic structures in the same graph and time. Each macroeconomic structure is formed by a 
large number of general structures, sub-structures and mini-structures on different axes, levels, and cubes by sizes and colors (see Figure 2). However, the detailed analysis of each structure by axes, levels, perimeters and cubes by sizes and colors depends on the parameters established by our research. Finally, all these general structures, sub-structures and mini-structures utilize the global structural imbalance principle under the application of the Omnia Mobilis assumption (Ruiz Estrada, 2011).

\subsection{Introduction to the Cubes Cartesian Space (Cubes-Cartesian Space)}

The cubes Cartesian space (Cubes-Cartesian Space) is formed from an infinite number of general axes $\left(A_{0}, A_{1}, \ldots, A_{\infty}\right)$, where each axis exhibits different levels $\left(\mathrm{L}_{0}, \mathrm{~L}_{1}, \ldots, \mathrm{L}_{\infty}\right)$, perimeters $\left(\mathrm{P}_{0}, \mathrm{P}_{1}, \mathrm{P}_{2, \ldots,} \mathrm{P}_{\infty}\right)$, and cubes with different sizes and colors $\left(\mathrm{C}_{0 / \beta}, \mathrm{C}_{1 / \beta, \ldots,}, \mathrm{C}_{\infty / \beta}\right)$. Therefore, the coordinate system of the Cubes-Cartesian space is represented by $\mathrm{S}_{\mathrm{A}: \mathrm{L}: \mathrm{P}: \mathrm{C}}=\left(A_{i}, L_{,}, P_{k} C_{s / \beta}\right)$ (see for example Poincare $\&$ Halsted 1913). Similarly, the subscripts such as $i, j, k$ and $s$ stand for different values between 0 and $\infty$, and $\beta$ represents different colors of each cube in different levels $\left(\mathrm{L}_{0}\right.$, $\left.\mathrm{L}_{1}, \ldots, \mathrm{L}_{\infty}\right)$. All these cubes $\left(\mathrm{C}_{\mathrm{s} / \beta}\right)$ with different sizes and colors in the same axis under the same level $\left(\mathrm{L}_{0}, \mathrm{~L}_{1}, ., \mathrm{L}_{\infty}\right)$ and different perimeters $\left(\mathrm{P}_{0}, \mathrm{P}_{1}, \mathrm{P}_{2}, \ldots, \mathrm{P}_{\infty}\right)$ would be joined together based on the application of a concept called the links structures that is represented by the symbol “@.” Moreover, the Cubes-Cartesian space coordinate system is shown by the following expression 1 and Figure 2:

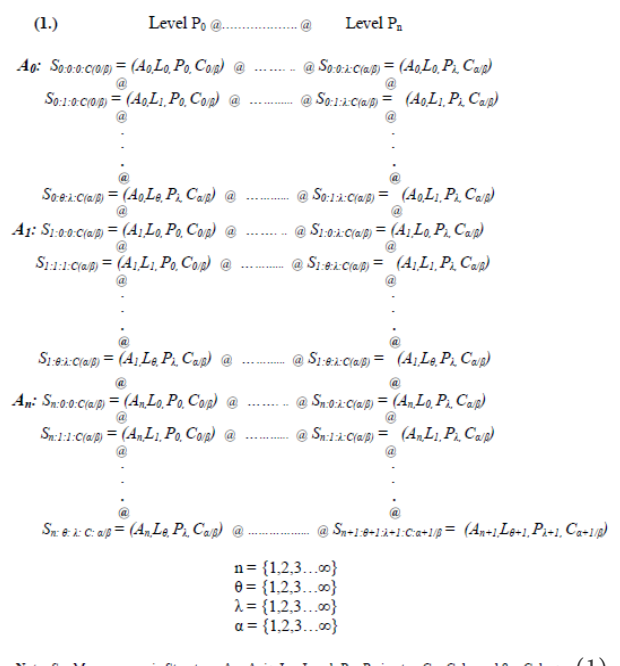

Finally, the Cubes-Cartesian space presents a general function $\mathrm{Yg}$ that is obtained from the interconnection of all macroeconomic structures $\left(\mathrm{S}_{0}, \mathrm{~S}_{1}, \ldots, \mathrm{S}_{\mathrm{n}}\right)$ under different axes $\left(A_{1}, A_{2}, \ldots, A_{n}\right)$, levels $\left(L_{1}, L_{2}, \ldots, L_{n}\right)$, perimeters $\left(\mathrm{P}_{0}, \mathrm{P}_{1}, \mathrm{P}_{2, \ldots,} \mathrm{P}_{\mathrm{n}}\right)$ and Cubes with different sizes and colors $\left(\mathrm{C}_{0 / \beta}, \mathrm{C}_{1 / \beta, \ldots}, \mathrm{C}_{\mathrm{n} / \mathrm{\beta}}\right)$, as highlighted in Expression 2.

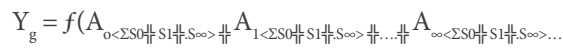

Note: Yg = The General Variable, 岑= Interconnection, $A_{i}=$ Axis and $S_{i}=$ General Structures.

However, the size of all cubes in terms of level is under the use of parameters that we establish in our research. The parameters we fix in this model directly depend on the quantity of money or number of units. The changes of all cubes size depend on the constant changes in the volume of money or the number of units between $\mathrm{n}$-periods of time. However, if we assume that all the cubes in different levels are changing constantly in real time, then all the cubes can experience an expansion, contraction or stagnation. We propose that all the cubes in level zero (mini-structures) are smaller than the cubes in level one (sub-structures), and finally, the cubes in level one (sub-structures) are smaller than the cubes in level two (general structure), as depicted in Figure 4 and Figure 5.

\subsection{The Process of Forecasting into the} Macroeconomics Structures Vulnerability Analysis (MSV-Analysis)

The process of forecasting in the Macroeconomics Structures Vulnerability Analysis (MSV-Analysis) assumes n-number of vectors. To forecast Yg, we suggest in our model the predicted value of the Yg to be equal to the interconnection of n-number of Sub-Y... Ys. We assume, therefore, two types of time in the process of the forecasting Macroeconomics Structures Vulnerability Analysis (MSV Analysis). It is followed by the general time speed (-gt) running in Yg and the partial times speed (-pt), which is running in each sub-Y. Hence, the general time speed ( the synchronization of all partial time speeds ( the macroeconomics structures vulnerability analysis (MSV-Analysis). Different partial time speeds ( run in different magnitudes of time in each level of analysis. In our case, we work on three different levels 
Cube- 1 Level cero $0 \geq \mathrm{TV}_{\mathrm{i}} \leq \mathrm{V}_{1}$ Minimum value

Cube-2 Level one $\mathrm{V}_{1} \geq \mathrm{TV}_{\mathrm{i}} \leq \mathrm{V}_{2}$ Medium value

Cube-3 Level two $V_{2} \geq \mathrm{TV}_{\mathrm{i}} \leq \mathrm{V}_{3}$ Large value

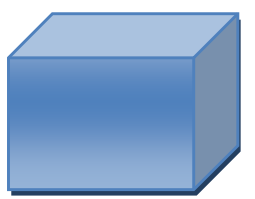

Cube-n Level-n $V_{3} \geq T V_{n} \leq V_{n}$ Extra-large value

Note: $\mathrm{TV}=$ Total Value, $\mathrm{V}=$ Value and. $\mathrm{n}$. is equal to any value between 1 and $\infty$.

Figure 4. Cubes Parameters

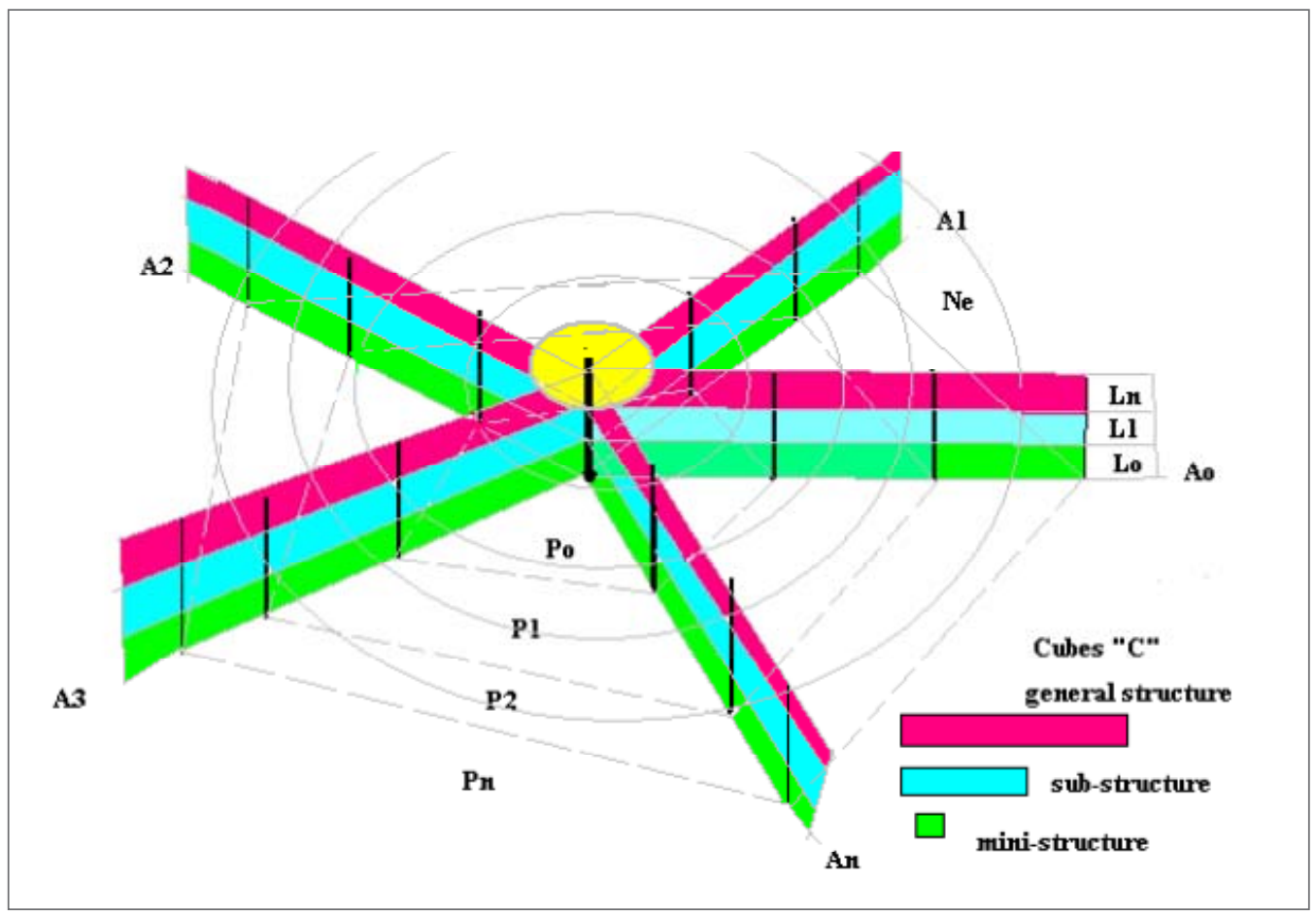

Figure 5. The Cubes-Cartesian Space Coordinate System 
of analysis, from level 0 to level 2. The partial times speeds (-pt) also depend on different axes and perimeter levels in the macroeconomics structures vulnerability analysis (MSV-Analysis). The first stage of the forecasting condition in the macroeconomics structures vulnerability analysis (MSV-Analysis) is simplified with the help of Expression 2.

Finally, we reach the general function, which is presented by Expression 1:

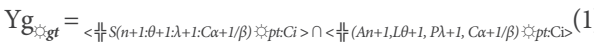

Note: $\mathrm{GF}=$ General forecast point;

少= Interconnection;

$\mathrm{C}_{\mathrm{i}}=$ confidence interval;

pt = Partial Times Speed

and $=$ General Time Speed

The confidence interval for the different predicted values of $\mathrm{C}_{\mathrm{i}}$ (see Expression 5) depend on the general time speed ( degrees of freedom, and the expression multiplied by - $\mathrm{gt} / \mathrm{n}$ is the standard error of prediction using the estimated standard deviation of the stochastic term deviation of the stochastic term $\hat{S}_{\mathrm{i}}$. Nevertheless, the forecast interval (Bertrand, Duflo, \& Mullainathan, 2004) is open according to each axis, perimeter and level in the macroeconomics structures vulnerability analysis (MSV-Analysis) that predicts the Yg. It is based on the interconnection of large number of sub-Ys because we assume that the space (or world) is Multi-Dimensional (Fedorchuk, Levin, \& Shchepin, 1999), and different sub-spaces in the same space are moving with different speeds of time. Hence, we assume that there exists a general time speed (-gt) under the synchronization of infinity partial times speed (tht). Finally, the forecasting process in the macroeconomics structures vulnerability analysis (MSV-Analysis) exhibits a different perspective to understand the future behavior of complex economic scenarios from a multi-dimensional perspective; the idea about short, medium and long run is totally different from the classical conception of time in this research paper.

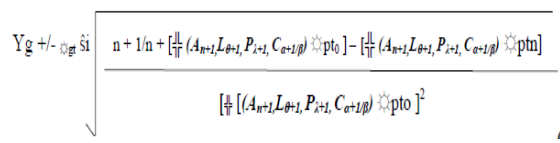

Note: $\mathrm{A}=$ Axis; $\mathrm{L}=$ Level; $\mathrm{P}=$ Perimeter; $\mathrm{C}=$ Cube; $\beta=$ Colors; $;$ pt $=$ Partial Times Speed and $\mathrm{gt}=$ General Time Speed

\section{The Mega-Economic Surface Interactive System}

\subsection{Introduction}

The literature demonstrates that in the study of any economy from a microeconomic or macroeconomic perspective, it is common to observe the use of static or dynamic economic models under the application of partial (Cournot, 1897) and general equilibrium (Hicks, 1939). These models explain and analyze the behavior of complex structural economic problems of any economy under ex ante or ex post results (McClelland, 1975). The results are calculated using sophisticated and complex econometrics models together with use of advanced software and computer solutions. More to the point, all of these results were plotted on the two-dimensional Cartesian plane (X,Y). We came to the conclusion from the brief survey of the literature that until today any multi-dimensional graphical modeling that can permit the observation of a large number of variables fixed in the same graphical space and interacting simultaneously in real time is not available. Therefore, we propose a multi-dimensional graphical modeling under the application of the MEISurface. The MEI-Surface can facilitate the visualization of a large number of macroeconomics variables in constant movement under the same physical space; with it, one can observe the fast and abrupt changes of all macroeconomics variables of any economy in real time. The MIE-Surface is available to alert policy makers, central banks, government agencies, the private sector and investors about a possible economic boom or recession at an early stage. Early warnings would help policy makers across the globe to prevent their economies from unwanted situations by implementing and executing appropriate policies in a timely manner.

\subsection{The Mega-Economic Surface Interactive System}

This section is aimed to explaining in detail the construction of the Mega-economic Interactive Surface (MIE-Surface). In the first instance, the MIE-Surface is built by an infinite number of axes. Each axis is represented by a vertical line, and the vertical line presents 
positive and negative real numbers (see Figure 1). The first axis starts from $\mathrm{X} 11$, until we jump to $\mathrm{X} \infty \infty_{\text {... (see }}$ Figure 6). Hence, each axis in the MIE-Surface represents an independent variable. At the same time, the MIE-Surface can plot only one point on each axis. We are considering the growth rates of variables to generate the real time effect on each axis. The MIE-Surface needs to join each growth rate result of each axis to its neighbor growth rate results using a straight line. However, it does not mean that they are dependent. The bottom line is builds a single surface based on the linking of all axes together in the same physical space. The MIE-Surface is fixed in the infinity box coordinate system (see Figure 6). The infinity box coordinate system is a huge cube formed by combining infinite vertical axes. The infinite vertical axes present positive and negative coordinates. All positive and negative values are located on the top and bottom of the cube, respectively. We assume that geometrically the number zero " 0 " is not a single value or number located in the center of the two-dimensional and 3-dimensional Cartesian plane. Our point of view is that the number zero " 0 " is a large and infinite surface that is fixed at the middle part of the infinity box coordinate system (see Figure 6). The MIE-Surface is not a single dependent variable. The single dependent variable is represented through a large and single surface that is moving in real time in the same physical space. In fact, the application of the Omnia Mobilis assumption (Ruiz Estrada, 2011) is a basic condition to generate a real time effect on the MIE-Surface. Hence, the study of any economy according to this research needs to be in real time because the economy of any country does not exhibit a static behavior. Every economy has a dynamic and multi-dimensional behavior. We need to assume that the economy of any country always remains in permanent transformation. The assumption about the behavior of an economy (dynamic and multi-dimensional) can be represented with the help of a large surface that is moving in real time in a multi-dimensional graphical space. Similarly, the analysis of the MEISurface depends on the position of the surface and is displayed into the infinity box coordinate system (see Figure 6). The position of the surface into the infinity box coordinate system can help to determinate the situation of any economy. Additionally, the position of MIE-Surface into the infinity box coordinates system can allocate a specific color to the surface (see Figure 6). For example, the economic stability surface presents a green color (See Expression 4), the economic stagnation surface a black color (see Expression 5), the economic recession alert surface a yellow color (see Expression 6), and finally, the economic crisis surface a red color (see Expression 7). An animation of the MEI-Surface basically needs the application of "global balance "and "real time" conditions.

\subsection{Model}

The MEI-Surface presents an alternative graphical and mathematical approach to analyze any economy from a multi-dimensional perspective. This new multidimensional graphical modeling can warn about the real situation of any economy immediately. The MIESurface is an alternative economic graphical modeling that could help policy makers, central banks, private and public sectors and investors in no time to take appropriate decisions in a timely fashion to build suitable macroeconomic policies (Keynes, 1936) and at the same time, to reduce the risks associated with portfolios, stock markets and foreign direct investment (FDI) (Issing, 2011). The Mega-economic interactive surface function follows:

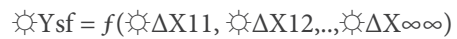

$=$ Real Time Ysf $=$ Surface $\Delta=$ Growth rate 3

Each value in each axis is based on measuring a growth rate as follows:

$\Delta \mathrm{X} 11=\Delta \mathrm{X} 11<\mathrm{t}+1>-\Delta \mathrm{X} 11<\mathrm{t} 0>\mathrm{x} 100 \%$

$\Delta \mathrm{X} 11<\mathrm{t} 0\rangle\langle\mathrm{t}+1\rangle=$ Future period of time $<\mathrm{to}>=$ Initial period of time

Therefore, the final mathematical structure to build the Mega-economic interactive Surface is based on expression (3):

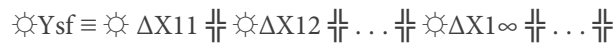

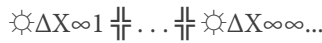
$\underline{t}=$ linkage of axes.

The MEI-Surface depends on the location of the surface in the infinity box coordinate system (see Figure 6). Hence, we have four possible results that can be clas- 


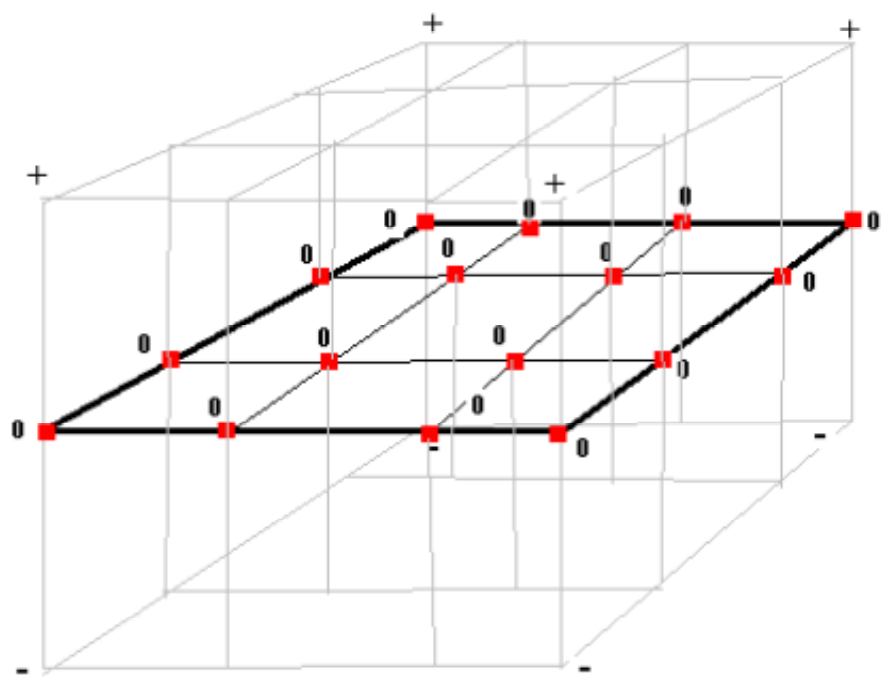

Figure 6. The Infinity Box Coordinate System

sified by economic stability (see Expression 4), economic stagnation (see Expression 5), economic recession alert (see Expression 6), and economic crisis (see Expression 7):

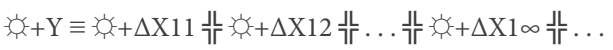

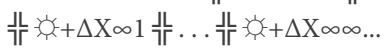

$\{$ if $\Delta \mathrm{Xij} \cap \mathrm{R}+$, then the surface becomes green $\equiv$ Economic Stability\}

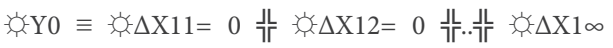
$=0$ 步. 少 if $\Delta \mathrm{Xij} \cap 0$, then the surface becomes black $\equiv$ Economic Stagnation\}

媇 $\Pi \mathrm{Y} \equiv$

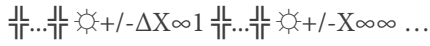

if $\Delta \mathrm{Xij} \cap \mathrm{R}+/$-, then the surface becomes yellow $\equiv$ Economic Recession Alert\}

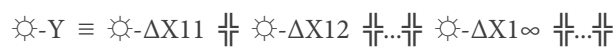
- $-\Delta \mathrm{X} \infty 1$ 少..尘 $-\Delta \mathrm{X} \infty \infty \ldots$

\{if $\Delta \mathrm{Xij} \cap \mathrm{R}$-, then the surface becomes red $\equiv$ Economic Crisis\}

\section{Conclusions}

This paper introduced four alternative experimental economic models to the macroeconomic literature to help researchers and policy makers to analyze and visualize macroeconomic scenarios from a multidimensional perspective. We have attempted to promote the uses of alternative analytical models by employing a new mathematical framework and multidimensional graphs that are based on the application of Econographicology. Furthermore, this paper sheds some light on the study of various possibilities to better understand macroeconomic scenarios to reduce the damage of possible economic failures (financial or economic crises) to the new generation of economists 
around the world. Policy makers and researchers, however, have to learn that economic failures cannot be avoided in all circumstances, but we can call an alert and reduce their damage with the help of real time economic modeling.

\section{References}

Balassa, B. (1985). Exports, Policy Choices and Economic Growth in Developing Countries after the 1973 Oil Shock. Journal of Development Economics, 18 (2), 23-35.

Barro, R. (1976). Rational expectations and the role of monetary policy. Journal of Monetary Economics, 2 (1), 1-32.

Bertrand, M., Duflo, E., \& Mullainathan, S. (2004). How Much Should We Trust Differences-in-Differences Estimates? Quarterly Journal of Economics, 119 (1), 249-275.

Brouwer, L. E. J. (1913). Über den natürlichen Dimensionsbegriff [About the Natural Notion of Dimension]. Journal für die reine und angewandte Mathematik, 1913 (142), 146-152.

Cournot, A. A., Bacon, N. T., \& Fisher, I. (1897). Researches into the Mathematical Principles of the Theory of Wealth ( $2^{\text {nd }}$ ed.). London, UK: Macmillan.

Edwards, S. (1993). Openness, Trade Liberalization, and Growth in Developing Countries. Journal of Economic Literature, 31 (3), 1358-1393.

Fedorchuk, V. V., Levin, M., Shchepin E. V. (1999). On Brouwer's Definition of Dimension. Russian Mathematical Surveys, 54 (2), 432-433.

Hansen, A. (1938). Full Recovery or Stagnation? New York: W.W. Norton \& Co. Inc.

Hicks, J. (1939). The Foundations of Welfare Economics. Economic Journal, 49 (196), 696-712.

Issing, O. (2011). The Crisis of European Monetary Union - Lessons to be Drawn. Journal of Policy Modeling, 33 (5), 737-749.

Inselberg, A., Dimsdale, B. (1994). Multidimensional Lines II: Proximity and Applications. SIAM Journal on Applied Mathematics, 54 (2), 578-596.

Keynes, J. M. (1936). The General Theory of Employment, Interest and Money. London, UK: Macmillan.

McClelland, P. D. (1975). Causal Explanation and Model Building in History, Economics, and the
New Economic History. Ithaca, N.Y.: Cornell University Press.

Poincare, H., Halsted, G. B. (1913). The Foundations of Sciences: Sciences and Hypothesis, the Value of Sciences, Sciences and Method. New York, NY: Sciences Press.

Ricardo, D. (1819). On the Principles of Political Economy and Taxation ( $2^{\text {nd }}$ ed.). London, UK: John Murray.

Ruiz Estrada, M. A. (2007). Econographicology. International Journal of Economics Research, 4 (1), 75-86.

Ruiz Estrada, M. A. (2008). Is the Market in Dynamic Imbalance State? International Journal of Economic Research, 5 (2), 35-46.

Ruiz Estrada, M. A. (2011). Policy Modeling: Definition, Classification, and Evaluation. Journal of Policy Modeling, 33 (4), 523-678.

Ruiz Estrada, M. A. (2013). Is It Possible to Apply Multidimensional Graphical Methods in the Teaching and Learning of Economics. Contemporary Journal, 7 (4), 123-138.

Russell, B. A. W. (1897). An Essay of the Foundations of Geometry. Cambridge, UK: Cambridge University Press.

Schlicht, E. (1985). Isolation and Aggregation in Economics. Berlin: Springer-Verlag.

Smith, A. (1904). An Inquiry into the Nature and Causes of the Wealth of the Nations ( $5^{\text {th }}$ ed.). London, UK: Methuen \& Co., Ltd. 